\title{
Multi-Level Orthogonal Hopping Rendezvous
}

\author{
Jens P. Elsner, Student Member, IEEE, Hosung Park, Student Member, IEEE, \\ Jeonghoon Mo, Member, IEEE, Friedrich K. Jondral, Senior Member, IEEE
}

\begin{abstract}
We propose a rendezvous scheme based on multilevel orthogonal hopping for multi-channel medium access control protocols. Its benefits compared to random hopping are higher throughput due to higher rendezvous probability. Furthermore, it offers improved fairness over other orthogonal hopping schemes.
\end{abstract}

\section{INTRODUCTION}

Multi-channel MAC protocols organize and distribute data traffic across multiple channels. Compared to single channel MAC protocols they offer increased throughput, especially in networks with a high number of nodes. A class of multichannel protocols are parallel rendezvous protocols such as $\mathrm{SSCH}$ [1] or McMAC [2], that allow simultaneous transmission agreements to improve performance: Each node has its own home channel hopping sequence, so that multiple connections can be negotiated at the same time ${ }^{1}$. Transmitters tune to the home channels of their potential receivers to establish a connection. In [3], Mo et al. showed that parallel rendezvous protocols can achieve higher throughput than single rendezvous protocols due to their better scalability. Different classes of multi-channel MAC protocols were analyzed, including parallel rendezvous protocols with a random hopping home channel strategy.

Our contribution with this letter is two-fold: First, we consider a hopping strategy called multi-level orthogonal hopping [4] as an alternative to random hopping [2], [3] in a multichannel MAC framework and show that its performance is superior to that of random hopping. Second, we present the first detailed evaluation of parallel rendezvous methods in multi-channel MACs, as home channel (receiver side) collisions were not studied in [3].

\section{PARAllel RendezVous Multi-CHANNEL MAC AND HOPPING STRATEGIES}

In parallel rendezvous multi-channel MACs, each node dwells in its home channel when idle. The home channels of nodes are known within a neighborhood, e.g., by attaching a random seed to each packet that is sent [2]. The home channel of a device can be fixed or hopping based. The first approach is simple but may lead to unbalanced channel utilization unless home channels are carefully selected. Also, packet interception and jamming are comparatively easy. We thus limit our focus to synchronized packed based ("slow") home channel hopping strategies for parallel rendezvous MAC. They find application in networks where robustness and security are of great importance.

\footnotetext{
${ }^{1}$ In contrast, in single rendezvous MAC protocols, all nodes meet in a dedicated channel to negotiate transmissions.
}
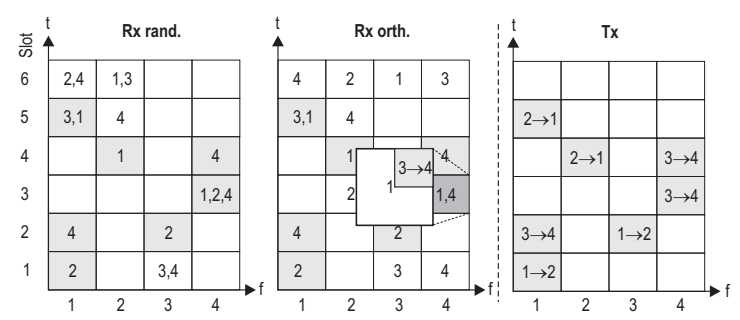

Fig. 1. Rx and Tx channel hopping for random and orthogonal rendezvous; $M=4, N=4$. The Tx plane shows on-going transmissions, e.g., $1 \rightarrow 2$ denotes a link from node 1 to node 2 . The Rx planes show a realization of home channels of idle nodes, overlaid with transmissions. We observe that, for orthogonal hopping, idle nodes do not collide on the Rx plane, but they can collide with on-going transmissions. E.g., in channel 4, slot 3 (drawn to perspective) the home channel of idle node 1 collides with " $3 \rightarrow 4$ ".

\section{A. Random hopping}

The simplest hopping strategy is random hopping in which the next home channel is determined by a pseudo-random sequence: Each device picks a seed to generate a different pseudo-random hopping sequence.

\section{B. Orthogonal hopping}

With orthogonal hopping, orthogonal sequences are used to minimize receiver side collisions. Two sequences $s=$ $\left(s_{1}, s_{2}, \ldots\right)$ and $s^{\prime}=\left(s_{1}^{\prime}, s_{2}^{\prime}, \ldots\right)$ are said to be orthogonal if $s_{i} \neq s_{i}^{\prime}$ for all $i=1,2, \ldots$ E.g., assume that the number of channels is $M=3$. Then, two hopping sequences $(1,3,2,1, \ldots)$ and $(3,2,1,2, \ldots)$ are orthogonal. Obviously, the maximum number of orthogonal sequences is $M$. When the number of devices $N$ is less than the number of channels, it is possible to keep the home channels collision-free. However, if $N>M$, home channels need to be shared. Fig. 1 shows a possible realization for the receive $(\mathrm{Rx})$ and transmit $(\mathrm{Tx})$ hopping sequences for $N=M=4$ with orthogonal ${ }^{2}$ and random hopping.

1) Orthogonal hopping with channel re-use: A straightforward solution to share home channels is assigning the same hopping sequence $s$ to two or more nodes. We evaluate channel re-use hopping for the purpose of comparison.

2) Multi-level orthogonal hopping: Another method to address the limited number of orthogonal codes is orthogonal hopping with multiple sets of orthogonal codes. Each of these sets can be seen as a layer, stacked up in multiple levels. Hence, a layer comprises $M$ sequences and any two sequences in a layer are orthogonal. E.g., for 5 nodes in 3 channels there

\footnotetext{
2"'Orthogonal" refers to the Rx (home) hopping sequences. The transmission hop sets are always orthogonal. For robust networks, as in Fig. 1, Tx hopping is used. McMAC [2] on the other hand does not employ Tx hopping; the performance model is the same in both cases.
} 
would be 2 layers, one layer with 3 nodes and another one with 2 nodes. Each layer has an associated pseudo-random sequence. To reduce correlation between layers, all sequences in a layer are pseudo-randomly permutated in every hop. In this way, collisions are avoided between nodes in the same layer - thus keeping the benefits of orthogonal hopping - while collisions between nodes in different layers are randomized.

\section{PERFORMANCE MODEL}

We use the discrete time Markov chain model of Mo et al. [3] to compare different hopping strategies. For the completeness of the paper, we briefly explain the model ${ }^{3}$. There are $N$ nodes that operate in $M$ channels. Nodes select communication partners uniformly and independently. In each time slot, an idle node tries to transmit a request-to-send to a chosen receiver with probability $p$; the parameter $p$ hence models the overall network load. If an agreement with the intended receiver is reached, a packet is transmitted over the following time slots. The packet lengths, given in number of slots, are assumed to be geometrically distributed with mean $1 / q$. This implies that each ongoing packet transmission ends independently with probability $q$ in each slot, allowing for a (memoryless) Markov chain model. The state space $\mathcal{S}$ of the chain comprises the number of currently active transmissions: $\mathcal{S}=\left\{0,1, \ldots, \min \left(\left\lfloor\frac{N}{2}\right\rfloor, M\right)\right\}$, where $\lfloor\cdot\rfloor$ denotes the floor function. The state changes either if new transmission agreements are made or if transmissions end. Let $S_{k}^{(i)}$ and $T_{k}^{(j)}$ denote the probability of $i$ transmissions starting and $j$ transmissions ending when the current state is $k$, respectively. The maximum number of transmissions that can end in the next time slot is $k$. The transition probability $p_{k, l}$ from state $k$ into state $l$ can then be written as

$$
p_{k, l}=\sum_{m=\max \{k-l, 0\}}^{k} S_{k}^{(m+l-k)} T_{k}^{(m)} .
$$

The $p_{k, l}$ are the elements of the stochastic transition probability matrix $P$. From $P$, one can calculate the steady state distribution vector $\pi$ by solving $\pi P=\pi$, i.e., by calculating the eigenvector of $P$ associated with eigenvalue 1 . Using the steady state distribution vector $\pi$, the total throughput $C$ can be calculated as ${ }^{4}$

$$
C=\sum_{i=0}^{|\mathcal{S}|-1} i \pi_{i}
$$

The termination probability $T_{k}^{(j)}$ can be easily computed from the assumption of independence: $T_{k}^{(j)}=\left(\begin{array}{c}k \\ j\end{array}\right) q^{j}(1-q)^{k-j}$. However, the (success) probability $S_{k}^{(j)}$ of $j$ new agreements is quite complicated for a parallel rendezvous protocol and is given by

$$
\begin{aligned}
S_{k}^{(j)}= & \sum_{a=0}^{N-2 k} \sum_{o=0}^{\min (M, a)} \sum_{i=0}^{o} P(A=a) P(O=o \mid A=a) \\
& P(I=i \mid A=a, O=o) P(J=j \mid A=a, I=i, O=o)
\end{aligned}
$$

\footnotetext{
${ }^{3}$ Confer [3] for further analysis and other relevant references.

${ }^{4}$ Note that this throughput measure includes the RTS/CTS messages, the practical data throughput will hence be less.
}

The conditional probabilities are calculated in the following. To have $j$ new connections, the following conditions need to be met: i) The number of nodes $A$ attempting to start a transmission should be equal to or larger than $j$, ii) the number of "one-attempt" channels $O$, i.e., the number of channels where exactly one device tries to initiate a transmission should be at least $j$, iii) the number of idle one-attempt channels $I$ should be larger than $j$ and iv) the number of idle receivers in an idle one-attempt channels $J$ should be exactly $j$.

The probability of $a$ idle nodes wanting to start a transmission given $k$ active nodes is $P(A=a)=\left(\begin{array}{c}N-2 k \\ a\end{array}\right) p^{a}(1-$ $p)^{N-2 k-a}$. The one-attempt probability $P(O=o \mid A=a)$ depends on the home channel hopping strategy and will be evaluated in the following for the different approaches. The probability that a one-attempt channel is idle is $P(I=$ $i \mid O=o, A=a)=\frac{\left(\begin{array}{c}k \\ o-i\end{array}\right)\left(\begin{array}{c}M-k \\ i\end{array}\right)}{\left(\begin{array}{c}M \\ o\end{array}\right)}$. Finally, the probability that a given transmitter finds its receiver is approximated by $p_{s}=\frac{N-2 k-a}{N-1}$ : We assume that all idle devices are equally busy, i.e., that no node is selected more often than others as a receiver. Accordingly, the probability that $j$ nodes find their receivers and can establish a new connection is $P(J=j \mid I=i, O=o, A=a)=\left(\begin{array}{l}i \\ j\end{array}\right) p_{s}^{j}\left(1-p_{s}\right)^{i-j}$.

\section{A. One-attempt probability}

The one-attempt probability or $P(O=o \mid A=a)$ models collisions in parallel rendezvous protocols and has a significant effect on the throughput $C$. Collisions occur if transmitters select i) the same receiver or ii) two different receivers with the same home channel. The latter probability differs depending on the home hopping strategies of devices and we would like to compare them for different strategies: random hopping and orthogonal hopping.

1) Random hopping: With random hopping, each node chooses its current home channel with equal probability from the number of available channels. Collisions can occur due to two events: Transmitters can select the same receiver or two selected receivers have the same home channel ${ }^{5}$. We calculate the one-attempt probability by conditioning on a certain selection of receivers and a certain selection of home channels.

The probability of a certain outcome $\vec{c}_{t}=\left(c_{1}, c_{2}, \ldots, c_{N}\right)$ of selected receivers is given by $P\left(\vec{C}_{t}=\vec{c}_{t} \mid A=a\right)=$ $\left(\begin{array}{c}a \\ c_{1}, c_{2}, \ldots, c_{N}\end{array}\right)\left(\frac{1}{N}\right)^{a}$, where $c_{i}$ denotes the number of transmitters choosing node $i$. Valid outcomes are all $\left(\begin{array}{c}N+a-1 \\ a\end{array}\right)$ $N$-compositions ${ }^{6}$ of $a$, i.e., $\sum_{i=1}^{N} c_{i}=a$, with $c_{i} \geq 0$.

Analogously, the probability of a certain outcome of receiver home channels $\vec{c}_{r}=\left(c_{1}, c_{2}, \ldots, c_{M}\right)$ is given by $P\left(\vec{C}_{r}=\vec{c}_{r} \mid A=a\right)=\left(\begin{array}{c}a \\ c_{1}, c_{2}, \ldots, c_{M}\end{array}\right)\left(\frac{1}{M}\right)^{a}$, where $c_{i}$ denotes the number of receivers dwelling in channel $i$. Valid outcomes are all $M$-compositions of $a$.

\footnotetext{
${ }^{5}$ The one-attempt probability given in [3] for the parallel rendezvous protocols is correct for orthogonal hopping and $N \leq M$. It gives an upper bound on the one-attempt probability for random hopping since the probability of receive channel collision is neglected. For orthogonal hopping and $N>M$, collisions of receive channels have to be taken into account as well.

${ }^{6}$ The compositions can be efficiently enumerated by the NEXTCOM algorithm described in [5, pp. 46].
} 
$\vec{c}_{r}$ and $\vec{c}_{t}$ denote the number of transmitters and receivers in a certain channel. For a given combination of $\vec{c}_{r}$ and $\vec{c}_{t}$, the number of one-attempts can be calculated by generating all receive channel assignments that yield the same $\vec{c}_{r}$ and counting the number of one-attempts when throwing balls into bins according to $\vec{c}_{t}$. Due to symmetry, this can easily be achieved by generating one possible receive channel assignment according to $\vec{c}_{r}$ and then generating all $a$ ! permutations of this assignment. The one-attempt probability $P\left(O=o \mid \vec{C}_{t}=\right.$ $\left.\vec{c}_{t}, \vec{C}_{r}=\vec{c}_{r}\right)$ is then calculated numerically by counting the number of outcomes with exactly $o$ one-attempts.

To calculate the overall unconditional one-attempt probability for random hopping, the probability of each transmit and receive channel combination needs to be considered:

$$
\begin{gathered}
P(O=o \mid A=a)=\sum_{i=1}^{\left(\begin{array}{c}
N+a-1 \\
a
\end{array}\right)} \sum_{j=1}^{\substack{M+a-1 \\
a}} P\left(\vec{C}_{t}=\vec{c}_{i}\right) P\left(\vec{C}_{r}=\vec{c}_{j}\right) \\
P\left(O=o \mid \vec{C}_{t}=\vec{c}_{t}, \vec{C}_{r}=\vec{c}_{r}\right),
\end{gathered}
$$

where the $\vec{c}_{i}$ and $\vec{c}_{j}$ run through all possible outcomes for transmitter and receiver collisions.

2) Orthogonal hopping: Orthogonal hopping assigns one of $M$ orthogonal home channels to nodes. If $N>M$, some nodes have to share a home channel. We distribute the available channels to nodes evenly so that the maximum of the number of nodes that have the same home channel is minimized. Let $L$ denote the number of full layers, i.e., $L=\left\lfloor\frac{N}{M}\right\rfloor$.

To describe the one-attempt probability, we need some auxiliary expressions. Let the number of bins with exactly one ball after throwing $x$ balls uniformly into $y$ bins be denoted by $B(y, x)$ and the number of bins with exactly zero balls as $Z(y, x)$. There is no closed-form solution for the probability distribution of $B(y, x)$ and $Z(y, x)$, but they can be calculated by considering a Markov chain, for which the states $\left(Y_{0}^{(n)}, Y_{1}^{(n)}\right)$ describe the number of bins containing zero balls $Y_{0}^{(n)}$ and exactly one ball $Y_{1}^{(n)}$ after $n$ throws ${ }^{7}$.

First, assume that $N \leq M$. The one-attempt probability $P(O=o \mid A=a)$ is then given by $P(B(M, a)=o)$.

Now, assume $N>M$. If $N \bmod M=0$, the one-attempt probability $P(O=o \mid A=a)$ is also given by $P(B(M, a)=$ $o$ ) due to symmetry, as all channels have equal probability of being selected. If $N \bmod M \neq 0, R=N \bmod M$ channels are used by $L+1$ nodes, while $M-R$ channels are used by $L$ nodes. The total one-attempt probability can be calculated by considering all possible outcomes of the distribution of attempts to the two partitions $R$ and $M-R$. The probability of having $k$ attempts in the $R$ bins is $\left(\begin{array}{l}a \\ k\end{array}\right) p_{R}^{k}\left(1-p_{R}\right)^{a-k}$ with $p_{R}=\frac{R(L+1)}{N}$.

${ }^{7}$ For this Markov chain, the initial state is $\left(Y_{0}^{(0)}, Y_{1}^{(0)}\right)=(N, 0)$ and the transition probabilities are $P\left(\left(Y_{0}^{(n+1)}, Y_{1}^{(n+1)}\right)=\right.$ $\left.\left(y_{0}, y_{1}\right) \mid\left(Y_{0}^{(n)}, Y_{1}^{(n)}\right)=\left(y_{0}, y_{1}\right)\right)=1-\frac{y_{0}+y_{1}}{N}, P\left(\left(Y_{0}^{(n+1)}, Y_{1}^{(n+1)}\right)=\right.$ $\left.\left(y_{0}-1, y_{1}+1\right) \mid\left(Y_{0}^{(n)}, Y_{1}^{(n)}\right)=\left(y_{0}, y_{1}\right)\right)=\frac{y_{0}}{N}$ and $P\left(\left(Y_{0}^{(n+1)}, Y_{1}^{(n+1)}\right)=\left(y_{0}, y_{1}-1\right) \mid\left(Y_{0}^{(n)}, Y_{1}^{(n)}\right)=\left(y_{0}, y_{1}\right)\right)=\frac{y_{1}}{N}$.
The one-attempt probability for orthogonal hopping is then

$$
\begin{aligned}
P(O=o \mid A=a)= & \sum_{o=1}^{a} \sum_{o_{1}, o_{2}} \sum_{k=0}^{a}\left(\begin{array}{l}
a \\
k
\end{array}\right) p_{R}^{k}\left(1-p_{R}\right)^{a-k} \\
& P\left(B(R, k)=o_{1}\right) \\
& P\left(B(M-R, a-k)=o_{2}\right),
\end{aligned}
$$

where second sum runs over all $\left(\begin{array}{c}o+1 \\ o\end{array}\right)=o+1$ possible 2-compositions of $o$ one-attempts.

\section{B. Single attempt collision probability}

The probability of collision $p_{c}$ gives the probability that a chosen single representative attempting node collides with one of the $a-1$ other attempting nodes. We will see that this probability depends on the home channel hopping strategy and that multi-level orthogonal hopping is fairer, as the variance of $p_{c}$ for different nodes is lower.

1) Random hopping: Consider two mutually exclusive events: i) at least one of $a-1$ attempting devices selects the representative node and ii) none of the $a-1$ attempting devices selects the representative node. In the first case, a collision occurs with probability 1 while in the second case, a collision only occurs if the home channel of another receiver is shared with the home channel of the representative node.

The probability of the first event is $1-\left(1-\frac{1}{N}\right)^{a-1}$, the second event has probability $\left(1-\frac{1}{N}\right)^{a-1}$. Let $1 \leq r \leq a-1$ denote the number of selected receivers out of $N-1$ potential receivers. The probability that $r$ unique receivers are selected is the same as $P(Z(n-1, a-1)=N-r-1)$, i.e., the probability that $N-r-1$ devices are not selected. By noting that at least one home channel must overlap for a collision, we have the collision probability $p_{c}=1-$ $\left(1-\frac{1}{N}\right)^{a-1}+\left(1-\frac{1}{N}\right)^{a-1} \sum_{r=1}^{a-1} P(Z(N-1, a-1)=N-$ $r-1)\left(1-\left(1-\frac{1}{M}\right)^{r}\right)$.

2) Orthogonal hopping with channel re-use: Here, some channels are occupied by $L=\left\lfloor\frac{N}{M}\right\rfloor$ nodes and the others by $L+1$ nodes. The collision probability $p_{c \text {,re-use differs depending }}$ on whether the representative node is in a channel with $L$ node or $L+1$ nodes. It is given by

$p_{c, \text { re-use }}= \begin{cases}p_{c, \text { re-use, high }}=1-\left(1-\frac{L}{N}\right)^{a-1}, & \text { with } L \text { devices; } \\ p_{c, \text { re-use, low }}=1-\left(1-\frac{L+1}{N}\right)^{a-1} & \text { otherwise }\end{cases}$

again assuming an even distribution of available channels to nodes so that the maximum of the number of nodes that have the same home channel is minimized. We see that the collision probability of some devices is higher than that of others in this scheme, which can be considered as being unfair.

3) Multi-level orthogonal hopping: In multi-level orthogonal hopping, the layers are scrambled to retain orthogonality within the layer but at the same time distribute the possible conflicts evenly over all nodes. Again, we assume that the layers are filled from the bottom. A node in a full layer will then experience a collision with the probability $p_{c, \mathrm{ML}}=$ $\frac{R}{M}\left(1-\left(1-\frac{L+1}{N}\right)^{a-1}\right)+\left(1-\frac{R}{M}\right)\left(1-\left(1-\frac{L}{N}\right)^{a-1}\right)$, and with probability $p_{c, \mathrm{ML}}=p_{c \text {,re-use }}$ if it is in the last layer. Here again, $R=N \bmod M$ and $L=\left\lfloor\frac{N}{M}\right\rfloor$. 


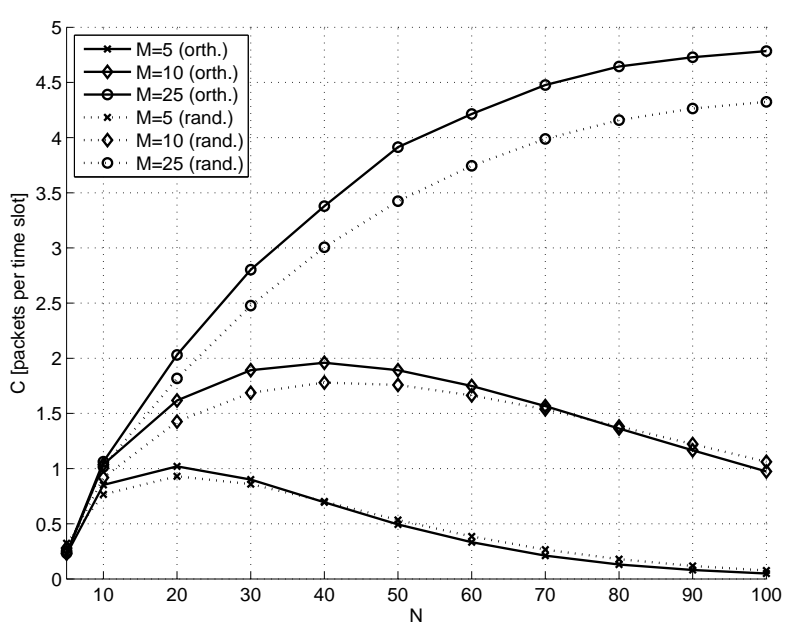

Fig. 2. Throughput versus number of nodes for $p=0.3, q=1$.

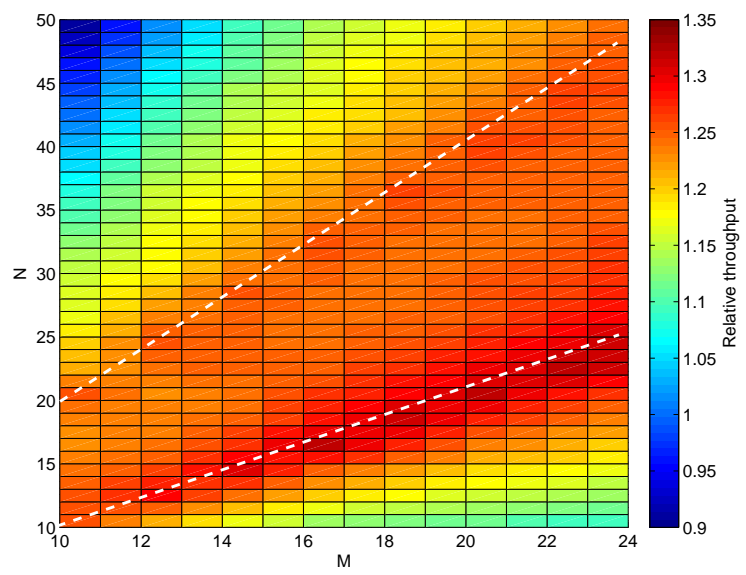

Fig. 3. Relative increase in throughput for $p=0.6, q=1$.

\section{NUMERICAL RESULTS}

We now evaluate the system model numerically and analyze the dependencies of various variables. The model closely approximates the performance of real implementations of parallel rendezvous MACs [2], [3].

\section{A. Throughput improvements}

Fig. 2 shows the total average throughput $C$ for orthogonal hopping and random hopping. A notable gain can be seen for a larger number of channels. For a fixed number of channels $M$ and a given network load $p$, there is a saturation point after which throughput decreases due to congestion. Interestingly, after saturation for large $N$ orthogonal hopping becomes slightly worse than random hopping. Orthogonal hopping distributes the transmission attempts evenly; therefore collisions happen in all channels. For random hopping there is a chance that nodes collide only in a few channels, leading to (slightly) higher throughput.

Fig. 3 shows the relative increase in throughput over $N$ and $M$. Also shown are lines indicating $N=M$ and $N=$ $2 M$. If $N=M$, the relative increase is maximal due to the fact that transmitter-side collisions have the greatest effect, for $N=2 M$ this effect is also visible. If the number of nodes significantly exceeds the number of channels and the network is operated in the congestion collapse regime, the gain

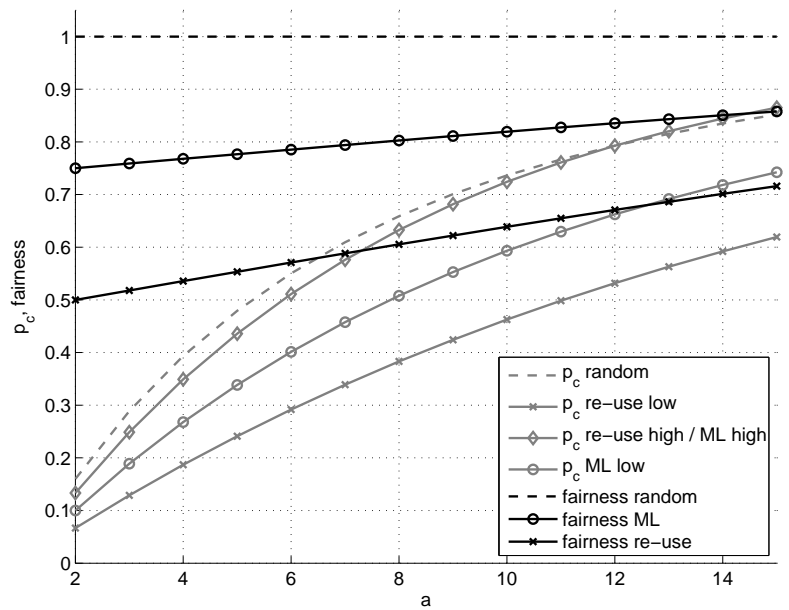

Fig. 4. Collision probability for a single node $p_{c}$ and fairness versus $a$ for different strategies, $M=10, N=15$.

eventually becomes smaller than 1 as was already observed in Fig. 2.

\section{B. Collision probability}

Fig. 4 shows the collision probabilities of a single connection as well as the fairness for each strategy. Fairness is defined as the ratio of the collision probabilities of nodes from a full layer to the collision probability of nodes from the last (nonfull) layer, i.e., $p_{c, \text { low }} / p_{c, \text { high }}$. For the re-use strategy, there are $R(L+1)$ nodes experiencing a higher probability of collision, whereas for the multi-level strategy this is the case for only $R$ nodes: The multi-layer hopping yields better fairness than the re-use strategy.

\section{CONCLUSION}

We evaluated a new rendezvous scheme based on multilevel orthogonal hopping for parallel rendezvous multi-channel MACs and compared its performance to other schemes, i.e., with random hopping and channel re-use hopping. Compared to those schemes, multi-level hopping offers increased throughput at better fairness.

The implementation costs of coordinated hopping are marginal since, in parallel rendezvous MACs, the hop sequences have to be communicated within a neighborhood anyway. Considering this, multi-level orthogonal hopping rendezvous can be beneficial in multi-channel MAC.

\section{REFERENCES}

[1] P. Bahl, R. Chandra, and J. Dunagan, "SSCH: slotted seeded channel hopping for capacity improvement in IEEE 802.11 ad-hoc wireless networks," in Proceedings of the 10th annual international conference on mobile computing and networking, ser. MobiCom '04. New York, NY, USA: ACM, 2004.

[2] W. H.-S. So, J. Walrand, and J. Mo, "McMAC: A Parallel Rendezvous Multi-Channel MAC Protocol," in IEEE WCNC, Mar 2007.

[3] J. Mo, H.-S. W. So, and J. Walrand, "Comparison of Multichannel MAC Protocols," IEEE Transactions on Mobile Computing, Jan 2008.

[4] J. P. Elsner, R. Tanbourgi, and F. Jondral, "Multiple Access Interference Mitigation Through Multi-Level Locally Orthogonal FH-CDMA ," in The 2011 Military Communications Conference, Baltimore, Maryland, USA, Nov. 2011.

[5] A. Nijenhuis and H. S. Wilf, Combinatorial Algorithms. Academic Press Inc., 1978. [Online]. Available: http://www.math.upenn.edu/ $\sim_{\text {wilf/ }}$ website/CombAlgDownld.html 